\title{
The Oral Microbiome and Lung Diseases
}

\author{
Chan Y. Pu ${ }^{1} \cdot$ Mukund Seshadri $^{2}$ - Sunita Manuballa ${ }^{2}$ Sai Yendamuri ${ }^{3,4}$ \\ Published online: 18 January 2020 \\ (C) Springer Nature Switzerland AG 2020
}

\begin{abstract}
Purpose of Review The understanding of the human microbiome, especially of the oral cavity, has expanded exponentially since the advent of 16 rRNA PCR gene sequencing. Since the respiratory tract starts from the oral cavity and ends in the lung, study of the relationship between the oral microbiota and the lungs will allow us to understand the changes in lung disease compared with healthy state.

Recent Findings The oral and lung microbiota were found to be similar, but the oral microbiota had greater diversity. The oral cavity especially the dorsal tongue was found to be a reservoir for bacteria causing pneumonia and chronic lung infection in cystic fibrosis. Oral and lung infections seem to all share the similar pathogenesis of oral microbiota dysbiosis. Certain oral bacteria were found to be potential biomarkers for lung cancer.

Summary Improvement in oral health is important especially in the management of lung diseases with infectious etiology. Oral microbiota can serve as biomarkers for diseases especially in lung cancer.
\end{abstract}

Keywords Oral microbiome $\cdot$ Lung $\cdot$ COPD $\cdot$ Cystic fibrosis $\cdot$ Pneumonia $\cdot$ Lung cancer

\section{Introduction and Overview}

The advent of next-generation sequencing has amplified our ability to assess the microbiome of different body niches as well as their alterations in pathological conditions. While the oral cavity and the lung are often viewed as distinct clinical entities, they are part of a continuum. This continuity is reflected in their microbiomes. Emerging evidence suggests

This article is part of the Topical Collection on Host Parasite Interactions in Periodontal Disease

Sai Yendamuri

sai.yendamuri@roswellpark.org

1 Pulmonary, Critical Care and Sleep Division, Department of Medicine, Jacobs School of Medicine and Biomedical Sciences, State University of New York at Buffalo, Buffalo, NY 14260, USA

2 Department of Oral Oncology, Roswell Park Comprehensive Cancer Center, Buffalo, NY, USA

3 Department of Thoracic Surgery, Roswell Park Cancer Institute, Roswell Park Comprehensive Cancer Center, Elm and Carlton Streets, Buffalo, NY 14263, USA

4 Department of Surgery, Jacobs School of Medicine and Biomedical Sciences, State University of New York at Buffalo, Buffalo, NY, USA that dysbiosis of the oral cavity is at the very least associated with and may impact the progression of several lung pathologies. The intent of this review is to summarize the studies that show this relationship. Data is summarized in sections separated by pathology, following a summary of factors influencing the microbiome of the oral cavity. Table 1 provides an "ata-glance" summary as well. We hope that this summary will be of use to the increasing number of investigators interested in this burgeoning field.

\section{Microbiome of the Oral Cavity}

As a system open to the environment, the oral cavity has a unique microbiome as it consists of mucosal surfaces that shed constantly as well as non-shedding surfaces of teeth. The oral microbiome is composed of a diverse ecological community of commensal, symbiotic, and pathogenic microorganisms that share the human oral cavity. The ecological niche consists of 5 distinct areas: teeth, saliva, tongue, gingival sulcus and periodontal pocket, and the remaining oral mucosa $[1,2]$. The abundant oral flora could not be fully identified previously because not all are cultivatable. But this changed with the advent of $16 \mathrm{~s}$ rRNA polymerase chain reaction (PCR) gene 

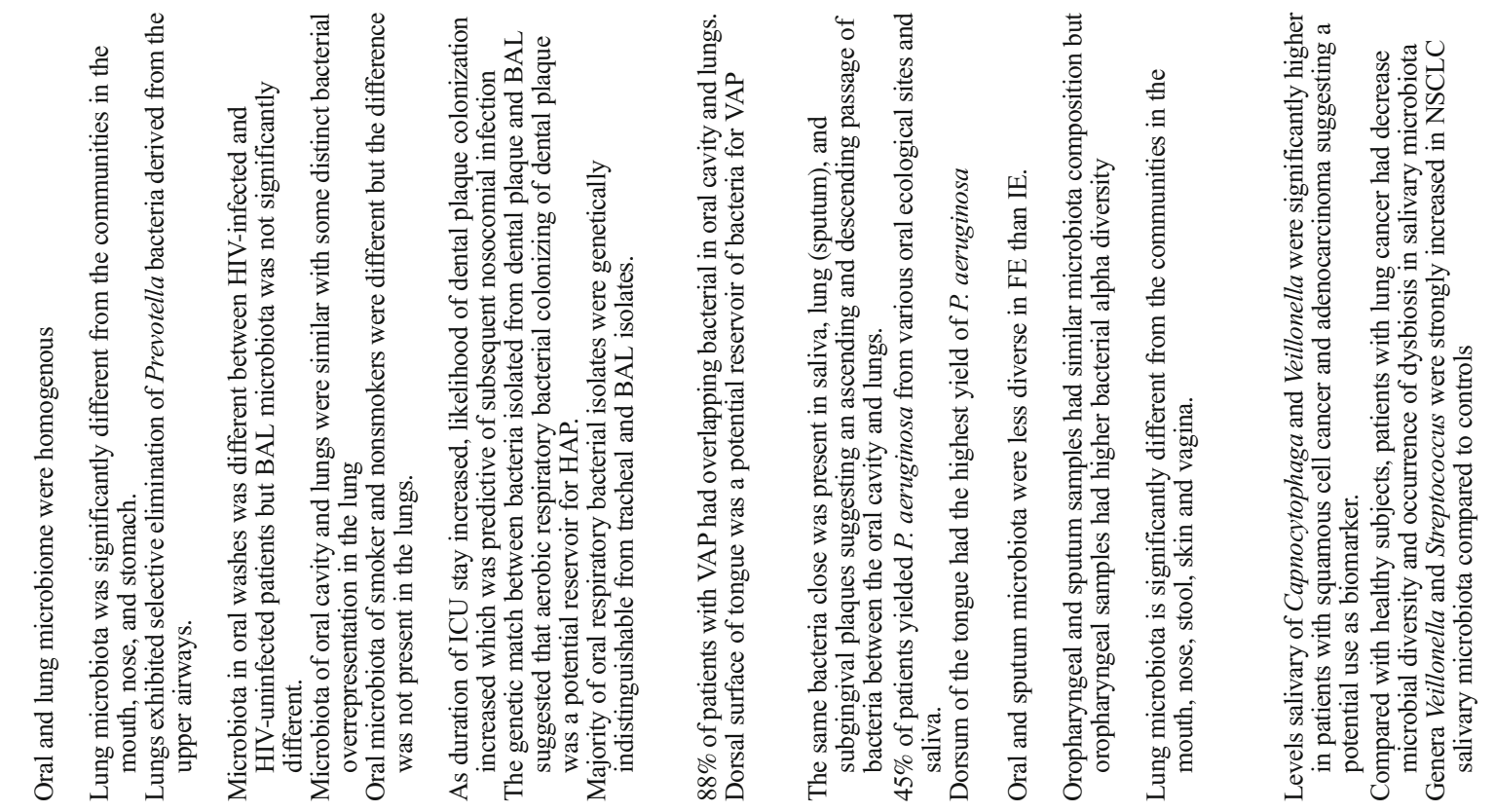

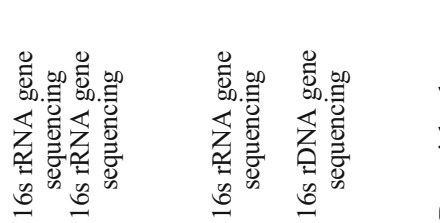

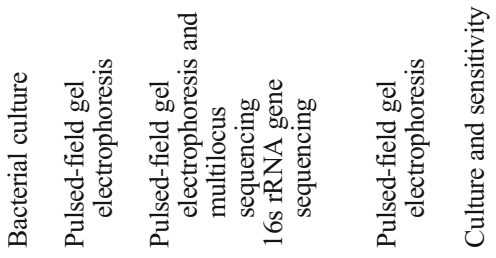
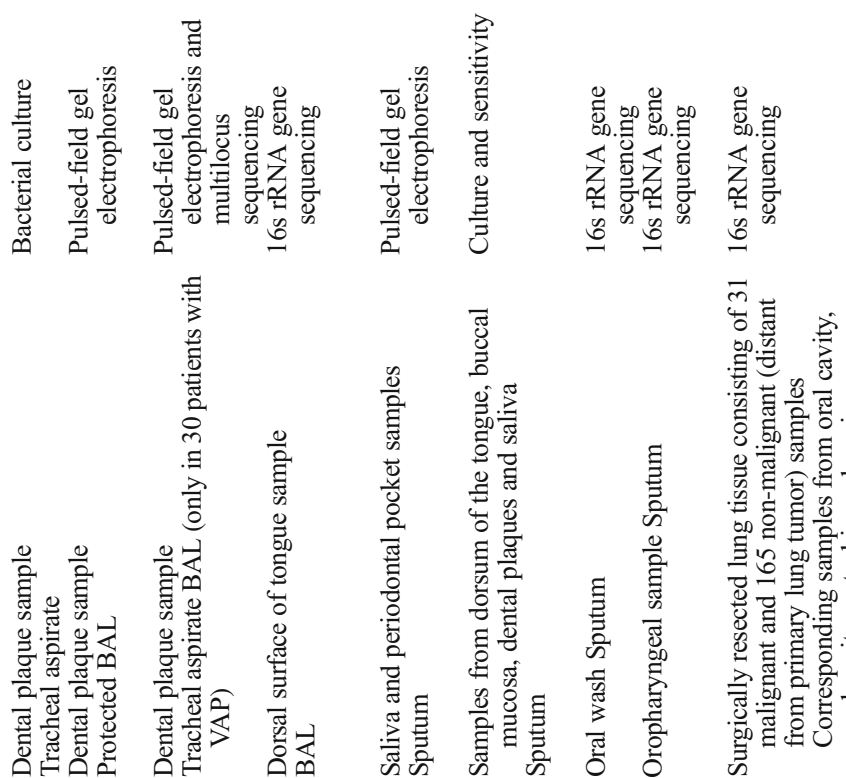

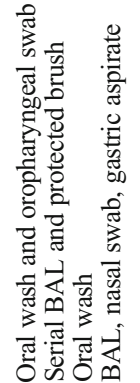
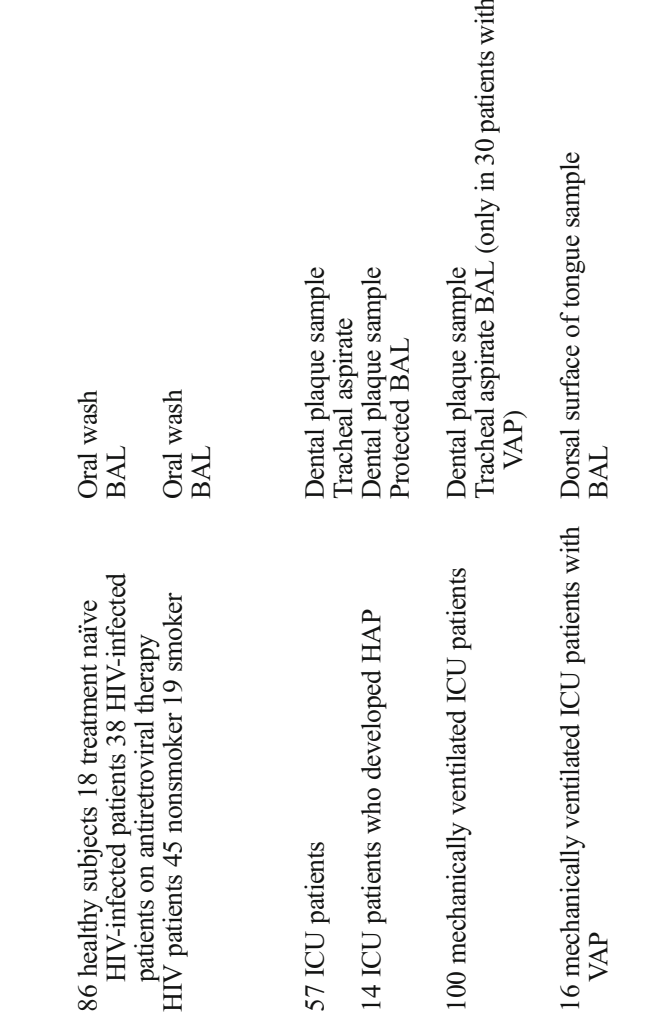

氖

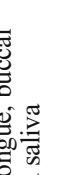
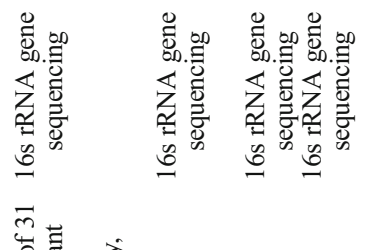

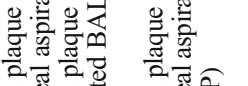

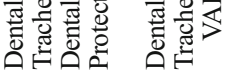
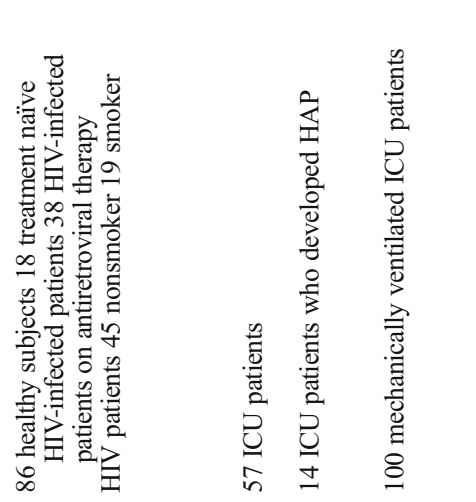

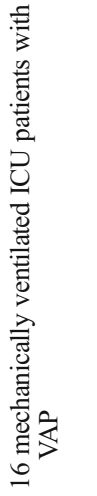

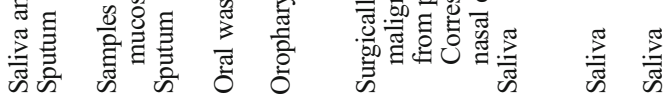
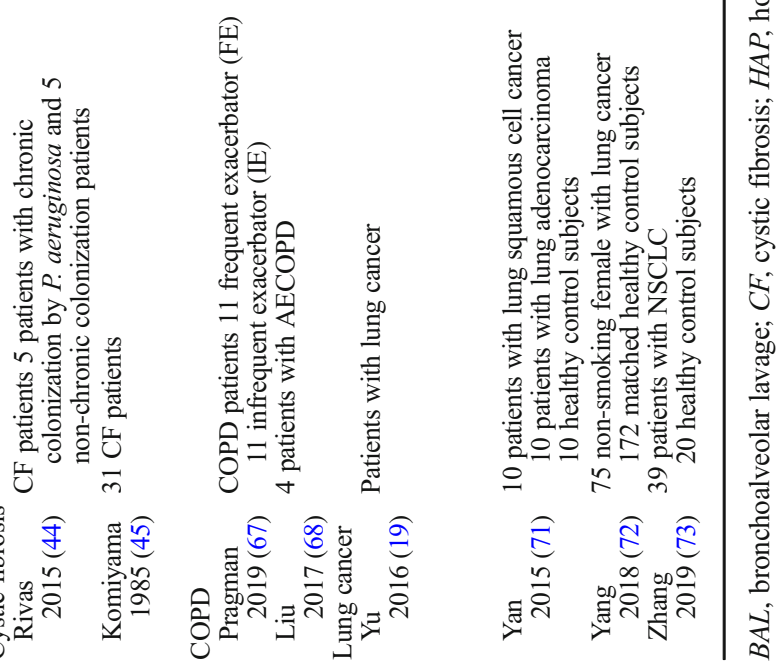
sequencing. The Human Oral Microbiome Database (HOMD) has a repository of bacterial genome sequences of the oral cavity containing about 800 species [3]. More recently, the expanded HOMD (eHOMD) was developed to include microbiome databases of the human aerodigestive tract as well [4]. The microbiome of saliva comes from bacteria shed from biofilms on oral tissues and does not have its own indigenous microbiota [5]. The most common microbiota of the oral cavity are Streptococcus, Lactobacillus, and Prevotella [2]. In the periodontal pocket, a shift in the abundance of low-abundance species has led to the "dysbiosis hypothesis" theorized to be a cause of periodontitis [6]. P. gingivalis has been suspected to be one of the key bacterial species underlying periodontitis. The "keystone" pathogen hypothesis describes the effect of a low-abundance microbial pathogen such as $P$. gingivalis that exerts a disproportionately large effect on their communities $[6,7]$. In the oral cavity, periodontitis is linked to $P$. gingivalis, which evolved to evade or circumvent the host immune system that triggers a destructive change in the normally homeostatic host-microbial interplay. In this manner, $P$. gingivalis acts as a keystone pathogen [6].

\section{Factors Influencing the Oral Microbiota}

The Lung HIV Microbiome Project studied the relationship between oral and lung microbiomes in HIV patients using bacterial 16S rRNA sequencing to compare the operational taxonomic units (OTUs) [8] between the two locations. Since HIV patients have impaired host defense, they are more vulnerable to infections, which may be reflected in their microbiota. Although the oral microbial populations were different in HIV-infected compared with HIV-uninfected patients, their bronchoalveolar lavage (BAL) microbial populations were not significantly different. CD4 cell counts did not correlate with the oral and lung microbiome on further analysis. Their lung microbiomes were mostly derived from their oral microbiome except for some unique bacterial such as Tropheryma whipplei. The use of antiretroviral therapy was associated with a reduced relative abundance of Tropheryma whipplei in the lungs of HIV-infected patients.

Ethnic background can determine salivary microbiota and can be altered with smoking $[9,10]$. Using the same cohort of HIV-uninfected patients from the Lung HIV Microbiome Project, oral microbiota was found to be different between smokers and nonsmokers but lung microbiota was not significantly altered by smoking [11]. Unfortunately, the author did not provide an explanation for this lack of difference. A study into the temporal shift in the oral microbiome found that communities remained stable over time in healthy subjects but community diversity varied between individuals [12].

\section{Relationship Between Oral and Lung Microbiota}

A study of microaspiration in healthy subjects using BAL samples showed that lower airway samples that are enriched with oral taxa (Prevotella or Veillonella) are associated with increased numbers of lymphocytes and neutrophils [13]. These taxa are also associated with a Th 17 lung inflammation phenotype [14]. Oral-derived microbiota such as Prevotella spp. is responsible for the regulation of pulmonary inflammatory responses shown by IL-17A in a mouse model [15]. In healthy subjects, as expected, the microbial biomass decreases from the oral cavity to the lungs but studies showed conflicting findings of whether oral and lung microbiome are similar or different. The topological continuity theory asserts that the respiratory tract from the nasal and oral cavities to the upper and lower airways are contiguous and the microbiota is indistinguishable between them [16]. This theory is supported by Charlson et al. who showed that the oral and lung microbiome are homogenous as the lungs contain bacterial sequences largely indistinguishable from the upper respiratory flora [17]. The countervailing theory is the island biogeography theory that describes the different human anatomic locations as different "islands" of habitation that differ in time and location [16]. A study by Bassis et al. showed that the oral and lung microbiome are found to be different in only half of the patients. Microaspiration was suggested as the cause of the similarity in the remaining half of the patients [18]. Risk of contamination by oral bacteria of the bronchoscope during sampling of BAL may also explain the differences. Since the studies evaluating oral and lung microbiota use bronchoscopy and BAL, evaluation of potential contamination was made by comparing the bacterial sequence and load of the serial BAL. Both authors were confident that carry over of oral contaminant was not an issue. Yu et al. overcame the potential oral contamination by comparing oral microbiota with lung microbiota (of normal lung tissue) obtained via surgical resection for lung cancer, which showed that the lung microbiome is different from the oral microbiome [19••]. Although the cohort of patients in the Yu et al. study had lung cancer, their non-malignant tissues were similar between patients and had greater phylogenetic diversity compared with malignant tissue which had low phylogenetic diversity and also showed different microbiota in different cancer histological diagnoses (adenocarcinoma vs squamous cell carcinoma).

\section{Dysbiosis in Acute Respiratory Tract Infections}

Poor oral hygiene has been linked to respiratory tract infection $[20,21]$. Dental plaque, which is a biofilm on tooth surfaces, 
has been identified as a reservoir of bacteria causing pneumonia [22-24]. Accumulation of dental plaque increased with ICU stay duration, which also increased the likelihood of colonization by aerobic pathogens (gram-negative rods first) [24]. This then led to nosocomial infections. Critical illness may allow more rapid dental plaque formation since these patients tend to have diminished salivation (xerostomia) and salivary $\mathrm{pH}$ [25]. Immigration of food-associated bacteria is reduced in critical illness when catabolic starvation state predominates due to reduced nutritional supply to commensal bacteria [26, 27]. Regular oral intake involves the ingestion of hard and fibrous food. This and the movement of tongue and cheeks during speech are absent in patients with critical illness especially if the patient is intubated [28]. Xerostomia due to stress of critical illness is worsened by lines and tubes traversing the oral airways which leaves the mouth open as well as medication that dries up secretions. The natural distribution of salivary immune factors such as IgA and lactoferrin is compromised in the setting of xerostomia [29]. The predominant bacterial species shift from gram-positive to gramnegative in the critical illness state $[27,30]$. The alteration in carbohydrates in buccal cells during critical illness has been shown to promote adherence of pathogenic bacteria to epithelial cells [31]. Benign Prevotella spp. and Veillonella spp. population [32] are displaced by potentially pathogenic bacteria such as $P$. aeruginosa and $K$. pneumonia $[30,33]$. Colonization of the oral cavity was found to be greater in patients with teeth or wearing dentures (73\%) compared with that in edentulous patients (37.5\%) [34]. This suggests that non-shedding surfaces (teeth and dentures) favor bacterial colonization greater than shedding surfaces (mucosa). This was confirmed by more rigorous studies of respiratory pathogens isolated from the oral cavity of patients with pneumonia which matched the strains found in the lung via bronchoalveolar lavage $[35,36]$. Using bacterial floral analysis of 16s rRNA gene among patients with pneumonia with aspiration risk, oral streptococci were the most common bacterial phylotypes detected [37]. Another 16s rRNA gene PCR amplification study showed that $88 \%$ of ventilator-associated pneumonia (VAP) patients had overlapping pathogens in the oral cavity and the lungs and identification of new putative uncultivatable and unreported species in 56\% of patients [38]. The dorsum of the tongue was suggested as a potential reservoir of bacteria for VAP.

Several distinct pathogeneses of oral microbiota linked to pneumonia have been described $[39,40]$. Oral pathogens may cause pneumonia via (1) aspiration of oral pathogens; (2) modification of lung mucosal surfaces by aspirated periodontal disease-associated enzymes and cytokines allowing adhesion and colonization by pathogens; (3) destruction of salivary pellicles on pathogenic bacteria by periodontal diseaseassociated enzymes; (4) airborne translocation; and (5) systemic bacteremia from periodontal infections. Mucosal alteration of epithelial cells in the form of loss of fibronectin, which functions to promote bacterial adhesion, occurs in $P$. aeruginosa colonization. Due to the action of proteases, the loss of fibronectin leads to unmasking of mucosal surfaces for respiratory pathogen adhesins. Oral bacteria can also destroy salivary pellicles through the action of sialidase on sialic acid residuals. This decreases the ability of mucins in the saliva to clear pathogens such as $H$. influenza. Cytokine release due to oral bacteria from the gingival crevice can stimulate the respiratory cells to produce other cytokines that recruit inflammatory cells that release hydrolytic enzymes. These enzymes can damage the respiratory epithelium leading to increase susceptibility to colonization of pathogens. In support of the relevance of these findings, improving oral hygiene and cleansing in at risk patients (ICU patients and elderly) have been advocated to prevent the occurrence of aspiration pneumonia, hospital-acquired pneumonia (HAP), and VAP. The use of chlorhexidine mouthwash reduces the risk of developing VAP but did not show mortality benefit [41, 42]. However, all results examining this issue do not show consensus in the conclusions. A more recent meta-analysis on the use of chlorohexidine mouthwash found an increase in mortality [43] although the reason was unclear. Clearly, further work needs to be done in this area.

\section{Dysbiosis in Cystic Fibrosis}

Cystic fibrosis (CF) lung disease is characterized by chronic colonization of bacteria such as Haemophilus influenzae, Staphylococcus aureus, and Pseudomonas aeruginosa. The oral-lung axis is thought to be bidirectional in CF. A casecontrolled study showed genetic relatedness between subgingival plaque and lung $P$. aeruginosa $[44 \bullet \bullet]$. The oral cavity is suggested as a potential reservoir of $P$. aeruginosa allowing for initial colonization and subsequent recolonization of $P$. aeruginosa in CF patients. The ascent of pulmonary bacteria can also replete the oral bacterial reservoir. The dorsum of the tongue was found to be the most common location for P. aeruginosa colonization in the oral cavity [45]. Although normal healthy lungs have coordinated mucociliary clearance to limit bacteria migration into the lungs, CF patients are particularly vulnerable to bacterial migration into the lungs due to dysfunctional cilia caused by dense mucus [46] and impaired alveolar macrophages and autophagy [47, 48]. The use of sputum to reflect the lung microbiome was studied by Hogan et al. who showed that the most abundant pathogen in sputum reflects the predominant taxa identified from protected brushing of the lung [49]. Nonetheless, the diversity of lung microbiome is lower in the lung compared with sputum especially in advance CF [49-51]. Toothbrushes were found to be a potential reservoir of CF-associated bacteria such as $P$. aeruginosa, $S$. aureus, S. maltophilia, 
A. xylosoxidans, and S. marcescens [52]. CF bacteria on the toothbrush's bristles can be introduced from the environment into the patient or be a source of re-inoculation to the oral cavity of the host.

An interesting mechanistic basis for the influence of the oral microbiome on the lung microbiome was proposed in cystic fibrosis patients. Oral metabolites can travel passively to the lungs and affect the lung microbiome in CF patients through cross-feeding. 2,3-Butanedione is a byproduct of alternative fermentation pathway which produces a neutral $\mathrm{pH}$ and avoids the lethal acidification of low $\mathrm{pH}$ fermentation. The 2,3butanedione gas likely produced by oral Streptococcus spp. is volatile and easily travels through the airways into the lungs which acts as a substrate for phenazine production by P. aeruginosa in CF lungs [53]. Phenazines are redox-active pigments that can serve as alternative electron acceptor for metabolism in hypoxic biofilm subregions [54]. Through proteome analysis 2,3-butanedione production was linked to biofilm production and increases the virulence factor of $P$. aeruginosa [55].

\section{Dysbiosis in COPD}

Tooth loss, periodontal disease, poor dental care, and lack of oral health knowledge were found to be associated with higher risk of having COPD [56-58]. Worse dental hygiene was associated with more respiratory symptoms in COPD and greater number of teeth has a positive correlation with more respiratory symptoms and sputum production [59]. As described in the pneumonia section above, the presence of non-shedding surfaces such as teeth and dentures favors bacterial colonization [34]. Periodontitis and COPD are hypothesized to be linked because both have similar pathophysiology in terms of elevated circulating inflammatory cytokines and mediators such as C-reactive protein, interleukin-8, tumor necrosis factor- $\alpha$, and matrix metalloproteinase $[60,61]$. Periodontitis can act as an inflammatory reservoir [62]. The cytokines from a local inflammatory response of periodontitis can spill into the systemic circulation with subsequent inflammatory damage to distal organ such as the lung. Neutrophilic inflammation is characteristic of both COPD and periodontitis [60]. Nevertheless, the causal relationship between periodontal disease and COPD can be due to the confounding effect of smoking [63]. Smoking is a major risk factor for periodontal disease [64] and is the main cause of COPD.

The most current understanding of bacterial acute exacerbation of COPD (AECOPD) comes from study of the lung microbiota, which showed that acquisition of new strain of Haemophilus influenzae, Moraxella catarrhalis, or Streptococcus pneumonia is strongly associated with AECOPD $[65,66]$. A study comparing the oral and sputum microbiota (representing the lung microbiota) showed that frequent exacerbators ( $\geq 1$ exacerbations per year) had a lower alpha diversity in their sputum microbiota than sputum microbiota of infrequent exacerbators [67]. Nonetheless, oral wash samples did not differ between frequent and infrequent exacerbators. PERMANOVA analyses found clustering of microbiota based on oral hygiene status, COPD severity, anatomic site, inhaler corticosteroid use, and smoking. Investigation of the potential use of oropharyngeal swab as a surrogate for sputum in AECOPD found that oropharyngeal swab and sputum had similar microbiota composition but oropharyngeal samples had higher diversity [68]. With the use of deep sequencing, the investigation Wang et al. on the lung microbiome in COPD showed clustering of phyla to Proteobacteria, Firmicutes, and Bacteroidetes subgroups [69]. During AECOPD, there was overall reduction in microbial $\alpha$ diversity and increase in relative abundance of Proteobacteria and decrease in Firmicutes. Wang et al. also showed that corticosteroid treatment decreased microbial $\alpha$ diversity with an increase of Proteobacteria over Firmicutes. On the other hand, treatment with antibiotics created an opposite trend.

\section{Dysbiosis in Lung Cancer}

Although an association of oral microbiome and cancer risk has been found in pancreatic cancers [70], our understanding of the impact of the oral microbiome on lung cancer pathophysiology remains limited. Yan et al. were the first to demonstrate the association between salivary microbiota with lung cancer [71]. Using 16s sequencing, levels of Capnocytophaga and Veillonella were significantly higher in patients with squamous cell cancer and adenocarcinoma suggesting their levels as potential use as biomarkers for disease detection or classification. The AUC of ROC of Veillonella for squamous cell cancer and adenocarcinoma were 0.81 and 0.68 , respectively, and the AUC of Capnocytophaga for squamous cell cancer and adenocarcinoma was 0.79 and 0.81 , respectively. In a different study, excluding the effect of smoking, salivary microbiome among 75 non-smoking female patients with lung cancer compared with 172 matched healthy control found decreased microbial diversity and occurrence of dysbiosis in the lung cancer group [72]. Bacterial genera Blastomonas and Sphingomonas were found to be significantly higher in the oral microbiota of the lung cancer group while Acinetobacter and Streptococcus were higher in the control group. The study also found a positive correlation between immunocytochemistry markers TTF-1 and CK 7 with Enterobacteriaceae, and Napsin A with genus Blastomonas. 
A study of salivary dysbiosis showed that genera Veillonella and Streptococcus were strongly increased in NSCLC compared with controls [73]. The UniFrac distance was significantly different between the groups on principal coordinates analysis. It also showed cross links among salivary microbiota dysbiosis, systemic inflammatory markers, and predicted Kyoto Encyclopedia of Genes and Genomes (KEGG) pathways.

\section{Dysbiosis in Lung Transplant}

Oral pharyngeal microbiota in patients undergoing lung transplant showed severe dysbiosis in taxonomic composition and respiration phenotypes with reduced richness and diversity and increased facultative and reduced aerobic bacteria in the pretransplant stage in the setting of their end-stage lung disease [74]. In 6 weeks to 3 months post-transplant, the richness and diversity were intermediate between healthy and pre-transplant patients. By 6 months, the post-transplant patients' oral pharyngeal microbiota resembled that of pre-transplant patients. All post-transplant patients were on antimicrobial and immunosuppressive therapy, which may have affected the patients' microbiota. However, analysis of pre-transplant patients on these agents suggested that the dysbiosis is not driven by antimicrobial nor immunosuppressive therapy.

\section{Conclusion}

With the improvement in PCR sequencing tools available to investigators, we have a greater understanding of the oral microbiome diversity and its systemic effect especially on the lungs. The diversity of microbiota decreases as we descend from the oral cavity to the lungs. Dysbiosis of the oral microbiota is linked to oral infections and a number of lung diseases especially pneumonia, CF, COPD, and lung cancer. The oral cavity was found to be a reservoir of bacteria causing disease in the acute conditions (aspiration pneumonia, HAP, and VAP) and chronic disease (CF). In lung cancer, changes in oral microbiota can be a biomarker of disease. Future studies should explore the use of oral microbiota dysbiosis as biomarker of disease and the manipulation of oral microbiota therapeutically to change lung disease progression.

\section{Compliance with Ethical Standards}

Conflict of Interest The authors declare that they have no conflicts of interest.

Human and Animal Rights and Informed Consent This article does not contain any studies with human or animal subjects performed by any of the authors.

\section{References}

Papers of particular interest, published recently, have been highlighted as:

•- Of major importance

1. Aas JA, Paster BJ, Stokes LN, Olsen I, Dewhirst FE. Defining the normal bacterial flora of the oral cavity. J Clin Microbiol. 2005;43(11):5721-32.

2. Zaura E, Keijser BJ, Huse SM, Crielaard W. Defining the healthy "core microbiome" of oral microbial communities. BMC Microbiol. 2009;9(1):259.

3. Chen T, Yu W-H, Izard J, Baranova OV, Lakshmanan A, Dewhirst FE. The Human Oral Microbiome Database: a web accessible resource for investigating oral microbe taxonomic and genomic information. Database [Internet]. 2010 [cited 2019 Oct 2];2010. Available from: https://www.ncbi.nlm.nih.gov/pmc/articles/ PMC2911848/

4. Escapa IF, Chen T, Huang Y, Gajare P, Dewhirst FE, Lemon KP. New insights into human nostril microbiome from the Expanded Human Oral Microbiome Database (eHOMD): a resource for the microbiome of the human aerodigestive tract. Xu J, editor. mSystems [Internet]. 2018 [cited 2019 Nov 6];3(6). Available from: http://msystems.asm.org/lookup/doi/10.1128/mSystems. 00187-18

5. Costalonga M, Herzberg MC. The oral microbiome and the immunobiology of periodontal disease and caries. Immunol Lett. 2014;162(2 0 0):22-38.

6. Hajishengallis G, Liang S, Payne MA, Hashim A, Jotwani R, Eskan MA, et al. Low-abundance biofilm species orchestrates inflammatory periodontal disease through the commensal microbiota and complement. Cell Host Microbe. 2011;10(5):497-506.

7. Fisher CK, Mehta P. Identifying keystone species in the human gut microbiome from metagenomic timeseries using sparse linear regression. PLoS One [Internet]. 2014[cited 2019 Nov 6];9(7). Available from: https://www.ncbi.nlm.nih.gov/pmc/articles/ PMC4108331/

8. Beck JM, Schloss PD, Venkataraman A, Twigg H, Jablonski KA, Bushman FD, et al. Multicenter comparison of lung and oral microbiomes of HIV-infected and HIV-uninfected individuals. Am J Respir Crit Care Med. 2015;192(11):1335-44.

9. Mason MR, Nagaraja HN, Camerlengo T, Joshi V, Kumar PS. Deep sequencing identifies ethnicity-specific bacterial signatures in the oral microbiome. PLoS One [Internet]. 2013 [cited 2019 Sep 15];8(10). Available from: https://www.ncbi.nlm.nih.gov/pmc/ articles/PMC3806732/

10. Wu J, Peters BA, Dominianni C, Zhang Y, Pei Z, Yang L, et al. Cigarette smoking and the oral microbiome in a large study of American adults. ISME J. 2016;10(10):2435-46.

11. Morris A, Beck JM, Schloss PD, Campbell TB, Crothers K, Curtis $\mathrm{JL}$, et al. Comparison of the respiratory microbiome in healthy nonsmokers and smokers. Am J Respir Crit Care Med. 2013;187(10):1067-75.

12. Hall MW, Singh N, Ng KF, Lam DK, Goldberg MB, Tenenbaum $\mathrm{HC}$, et al. Inter-personal diversity and temporal dynamics of dental, tongue, and salivary microbiota in the healthy oral cavity. $\mathrm{Npj}$ Biofilms Microbiomes Jan 26;3(1). Available from: http://www. nature.com/articles/s41522-016-0011-0

13. Segal LN, Alekseyenko AV, Clemente JC, Kulkarni R, Wu B, Chen $\mathrm{H}$, et al. Enrichment of lung microbiome with supraglottic taxa is associated with increased pulmonary inflammation. Microbiome. 2013;1:19.

14. Segal LN, Clemente JC, Tsay J-CJ, Koralov SB, Keller BC, Wu $\mathrm{BG}$, et al. Enrichment of the lung microbiome with oral taxa is 
associated with lung inflammation of a Th17 phenotype. Nat Microbiol. 2016;1:16031.

15. Huffnagle G, Dickson R, Lukacs N. The respiratory tract microbiome and lung inflammation: a two-way street. Mucosal Immunol. 2017;10(2):299-306.

16. Mammen MJ, Sethi S. COPD and the microbiome. Respirology. 2016;21(4):590-9.

17. Charlson ES, Bittinger K, Haas AR, Fitzgerald AS, Frank I, Yadav A, et al. Topographical continuity of bacterial populations in the healthy human respiratory tract. Am J Respir Crit Care Med. 2011;184(8):957-63.

18. Bassis CM, Erb-Downward JR, Dickson RP, Freeman CM, Schmidt TM, Young VB, et al. Analysis of the upper respiratory tract microbiotas as the source of the lung and gastric microbiotas in healthy individuals. mBio. 2015;6(2):e00037-15.

19.• Yu G, Gail MH, Consonni D, Carugno M, Humphrys M, Pesatori $\mathrm{AC}$, et al. Characterizing human lung tissue microbiota and its relationship to epidemiological and clinical features. Genome Biol [internet]. 2016 [cited 2019 Sep 23];17. Available from: https:// www.ncbi.nlm.nih.gov/pmc/articles/PMC4964003/. This study obtained lung microbiota surgical for comparison with oral microbiota without the risk of oral contamination which can create false position correlation.

20. Mojon P, Budtz-Jorgensen E, Michel J-P, Limeback H. Oral health and history of respiratory tract infection in frail institutionalised elders. Gerodontology. 1997;14(1):9-16.

21. Bágyi K, Haczku A, Márton I, Szabó J, Gáspár A, Andrási M, et al. Role of pathogenic oral flora in postoperative pneumonia following brain surgery. BMC Infect Dis. 2009;9:104.

22. Scannapieco FA, Stewart EM, Mylotte JM. Colonization of dental plaque by respiratory pathogens in medical intensive care patients. Crit Care Med. 1992;20(6):740-5.

23. Russell SL, Boylan RJ, Kaslick RS, Scannapieco FA, Katz RV. Respiratory pathogen colonization of the dental plaque of institutionalized elders. Spec Care Dentist. 1999;19(3):128-34.

24. Fourrier F, Duvivier B, Boutigny H, Roussel-Delvallez M, Chopin C. Colonization of dental plaque: a source of nosocomial infections in intensive care unit patients. Crit Care Med. 1998;26(2):301-8.

25. Ayars GH, Altman LC, Fretwell MD. Effect of decreased salivation and $\mathrm{pH}$ on the adherence of Klebsiella species to human buccal epithelial cells. Infect Immun. 1982;38(1):179-82.

26. David LA, Maurice CF, Carmody RN, Gootenberg DB, Button JE, Wolfe BE, et al. Diet rapidly and reproducibly alters the human gut microbiome. Nature. 2014;505(7484):559-63.

27. Dickson RP. The microbiome and critical illness. Lancet Respir Med. 2016;4(1):59-72.

28. Amaral SM, Cortês AQ, Pires FR. Nosocomial pneumonia: importance of the oral environment. J Bras Pneumol. 2009;35(11):1116 24.

29. Takei T, Aono W, Nagashima S, Yoshida T, Hashida T, Sobue S, et al. Change of salivary IgA secretion and caries development in irradiated rats. J Dent Res. 1994;73(9):1503-8.

30. Munro CL, Grap MJ. Oral health and care in the intensive care unit: state of the science. Am J Crit Care. 2004;13(1):25-34.

31. Weinmeister KD, Dal Nogare AR. Buccal cell carbohydrates are altered during critical illness. Am J Respir Crit Care Med. 1994;150(1):131-4.

32. Dickson RP, Erb-Downward JR, Freeman CM, McCloskey L, Beck JM, Huffnagle GB, et al. Spatial variation in the healthy human lung microbiome and the adapted island model of lung biogeography. Ann Am Thorac Soc. 2015;12(6):821-30.

33. Johanson WG, Pierce AK, Sanford JP. Changing pharyngeal bacterial flora of hospitalized patients. N Engl J Med. 1969;281(21): $1137-40$.
34. Scannapieco FA, Mylotte JM. Relationships between periodontal disease and bacterial pneumonia. J Periodontol. 1996;67(10s): 1114-22.

35. Heo S-M, Haase EM, Lesse AJ, Gill SR, Scannapieco FA. Genetic relationships between respiratory pathogens isolated from dental plaque and bronchoalveolar lavage fluid from patients in the intensive care unit undergoing mechanical ventilation. Clin Infect Dis. 2008;47(12):1562-70.

36. El-Solh AA, Pietrantoni C, Bhat A, Okada M, Zambon J, Aquilina A, et al. Colonization of dental plaques. Chest. 2004;126(5):157582.

37. Akata K, Yatera K, Yamasaki K, Kawanami T, Naito K, Noguchi S, et al. The significance of oral streptococci in patients with pneumonia with risk factors for aspiration: the bacterial floral analysis of $16 \mathrm{~S}$ ribosomal RNA gene using bronchoalveolar lavage fluid. BMC Pulm Med [Internet]. 2016 [cited 2019 Sep 15];16. Available from: https://www.ncbi.nlm.nih.gov/pmc/articles/ PMC4864928/

38. Bahrani-Mougeot FK, Paster BJ, Coleman S, Barbuto S, Brennan MT, Noll J, et al. Molecular analysis of oral and respiratory bacterial species associated with ventilator-associated pneumonia. J Clin Microbiol. 2007;45(5):1588-93.

39. Scannapieco FA. Role of Oral Bacteria in respiratory infection. J Periodontol. 1999;70(7):793-802.

40. Paju S, Scannapieco FA. Oral biofilms, periodontitis, and pulmonary infections. Oral Dis. 2007;13(6):508-12.

41. Zuckerman LM. Oral chlorhexidine use to prevent ventilatorassociated pneumonia in adults: review of the current literature. Dimens Crit Care Nurs. 2016;35(1):25-36.

42. Labeau SO, Van de Vyver K, Brusselaers N, Vogelaers D, Blot SI. Prevention of ventilator-associated pneumonia with oral antiseptics: a systematic review and meta-analysis. Lancet Infect Dis. 2011;11(11):845-54.

43. Price R, MacLennan G, Glen J. SuDDICU collaboration. Selective digestive or oropharyngeal decontamination and topical oropharyngeal chlorhexidine for prevention of death in general intensive care: systematic review and network meta-analysis. BMJ. 2014;348: g2197.

44.• Rivas Caldas R, Le Gall F, Revert K, Rault G, Virmaux M, Gouriou $\mathrm{S}$, et al. Pseudomonas aeruginosa and periodontal pathogens in the oral cavity and lungs of cystic fibrosis patients: a case-control study. J Clin Microbiol. 2015;53(6):1898-907 This study suggests the bidirectional relationship between the oral and lung axis.

45. Komiyama K, Tynan JJ, Habbick BF, Duncan DE, Liepert DJ. Pseudomonas aeruginosa in the oral cavity and sputum of patients with cystic fibrosis. Oral Surg Oral Med Oral Pathol. 1985;59(6): $590-4$.

46. Horváth G, Sorscher EJ. Luminal fluid tonicity regulates airway ciliary beating by altering membrane stretch and intracellular calcium. Cell Motil Cytoskeleton. 2008;65(6):469-75.

47. Simonin-Le Jeune K, Le Jeune A, Jouneau S, Belleguic C, Roux P$\mathrm{F}$, Jaguin $\mathrm{M}$, et al. Impaired functions of macrophage from cystic fibrosis patients: CD11b, TLR-5 decrease and SCD14, inflammatory cytokines increase. PLoS One [Internet]. 2013 [cited 2019 Sep 23];8(9). Available from: https://www.ncbi.nlm.nih.gov/pmc/ articles/PMC3787056/

48. Junkins RD, McCormick C, Lin T-J. The emerging potential of autophagy-based therapies in the treatment of cystic fibrosis lung infections. Autophagy. 2014;10(3):538-47.

49. Hogan DA, Willger SD, Dolben EL, Hampton TH, Stanton BA, Morrison HG, et al. Analysis of lung microbiota in bronchoalveolar lavage, protected brush and sputum samples from subjects with mild-to-moderate cystic fibrosis lung disease. PLoS One. 2016;11(3):e0149998.

50. Goddard AF, Staudinger BJ, Dowd SE, Joshi-Datar A, Wolcott RD, Aitken ML, et al. Direct sampling of cystic fibrosis lungs indicates 
that DNA-based analyses of upper-airway specimens can misrepresent lung microbiota. Proc Natl Acad Sci U S A. 2012;109(34): 13769-74.

51. Mayer ML, Blohmke CJ, Falsafi R, Fjell CD, Madera L, Turvey $\mathrm{SE}$, et al. Rescue of dysfunctional autophagy attenuates hyperinflammatory responses from cystic fibrosis cells. J Immunol. 2013;190(3):1227-38.

52. Passarelli Mantovani R, Sandri A, Boaretti M, Grilli A, Volpi S, Melotti P, et al. Toothbrushes may convey bacteria to the cystic fibrosis lower airways. J Oral Microbiol [Internet]. 2019 [cited 2019 Nov 6];11(1). Available from: https://www.ncbi.nlm.nih. gov/pmc/articles/PMC6713191/

53. Whiteson KL, Meinardi S, Lim YW, Schmieder R, Maughan H, Quinn R, et al. Breath gas metabolites and bacterial metagenomes from cystic fibrosis airways indicate active $\mathrm{pH}$ neutral 2,3butanedione fermentation. ISME J. 2014;8(6):1247-58.

54. Schiessl KT, Hu F, Jo J, Nazia SZ, Wang B, Price-Whelan A, et al. Phenazine production promotes antibiotic tolerance and metabolic heterogeneity in Pseudomonas aeruginosa biofilms. Nat Commun. 2019;10(1):1-10.

55. Venkataraman A, Rosenbaum MA, Werner JJ, Winans SC, Angenent LT. Metabolite transfer with the fermentation product 2,3-butanediol enhances virulence by Pseudomonas aeruginosa. ISME J. 2014;8(6):1210-20.

56. Wang Z, Zhou X, Zhang J, Zhang L, Song Y, Hu FB, et al. Periodontal health, oral health behaviours, and chronic obstructive pulmonary disease. J Clin Periodontol. 2009;36(9):750-5.

57. Cunningham TJ, Eke PI, Ford ES, Agaku IT, Wheaton AG, Croft JB. Cigarette smoking, tooth loss, and chronic obstructive pulmonary disease: findings from the behavioral risk factor surveillance system. J Periodontol. 2016;87(4):385-94.

58. Zeng X-T, Tu M-L, Liu D-Y, Zheng D, Zhang J, Leng W. Periodontal disease and risk of chronic obstructive pulmonary disease: a meta-analysis of observational studies. PLoS One [Internet]. 2012 [cited 2019 Sep 28];7(10). Available from: https://www. ncbi.nlm.nih.gov/pmc/articles/PMC3477163/

59. Gaeckle NT, Heyman B, Criner AJ, Criner GJ. Markers of dental health correlate with daily respiratory symptoms in COPD. Chronic Obstr Pulm Dis. 5(2):97-105.

60. Usher AK, Stockley RA. The link between chronic periodontitis and COPD: a common role for the neutrophil? BMC Med. 2013;11:241.

61. Barnes PJ, Celli BR. Chronic obstructive pulmonary disease: reasons for optimism. Therapy. 2009;6(6):769-70.

62. Hobbins S, Chapple IL, Sapey E, Stockley RA. Is periodontitis a comorbidity of COPD or can associations be explained by shared risk factors/behaviors? Int J Chron Obstruct Pulmon Dis. 2017;12: 1339-49.
63. Hyman JJ, Reid BC. Cigarette smoking, periodontal disease, and chronic obstructive pulmonary disease. J Periodontol. 2004;75(1): 7.

64. Tomar SL, Asma S. Smoking-attributable periodontitis in the United States: findings from NHANES III. J Periodontol. 2000;71(5):743-51.

65. Sethi S, Evans N, Grant BJB, Murphy TF. New strains of Bacteria and exacerbations of chronic obstructive pulmonary disease. $\mathrm{N}$ Engl J Med. 2002;347(7):465-71.

66. Sethi S, Wrona C, Eschberger K, Lobbins P, Cai X, Murphy TF. Inflammatory profile of new bacterial strain exacerbations of chronic obstructive pulmonary disease. Am J Respir Crit Care Med. 2008 Mar 1;177(5):491-7.

67. Pragman AA, Knutson KA, Gould TJ, Isaacson RE, Reilly CS, Wendt $\mathrm{CH}$. Chronic obstructive pulmonary disease upper airway microbiota alpha diversity is associated with exacerbation phenotype: a case-control observational study. Respir Res. 2019;20(1): 114.

68. Liu H-Y, Zhang S-Y, Yang W-Y, Su X-F, He Y, Zhou H-W, et al. Oropharyngeal and sputum microbiomes are similar following exacerbation of chronic obstructive pulmonary disease. Front Microbiol [Internet]. 2017 [cited 2019 Sep 29];8. Available from: https://www.frontiersin.org/articles/10.3389/fmicb.2017.01163/full

69. Wang Z, Bafadhel M, Haldar K, Spivak A, Mayhew D, Miller BE, et al. Lung microbiome dynamics in COPD exacerbations. Eur Respir J. 2016;47(4):1082-92.

70. Farrell JJ, Zhang L, Zhou H, Chia D, Elashoff D, Akin D, et al. Variations of oral microbiota are associated with pancreatic diseases including pancreatic cancer. Gut. 2012;61(4):582-8.

71. Yan X, Yang M, Liu J, Gao R, Hu J, Li J, et al. Discovery and validation of potential bacterial biomarkers for lung cancer. Am J Cancer Res. 2015;5(10):3111-22.

72. Yang J, Mu X, Wang Y, Zhu D, Zhang J, Liang C, et al. Dysbiosis of the salivary microbiome is associated with non-smoking female lung cancer and correlated with immunocytochemistry markers. Front Oncol [Internet]. 2018 [cited 2019 Sep 15];8. Available from: https://www.ncbi.nlm.nih.gov/pmc/articles/PMC6256243/

73. Zhang W, Luo J, Dong X, Zhao S, Hao Y, Peng C, et al. Salivary microbial dysbiosis is associated with systemic inflammatory markers and predicted oral metabolites in non-small cell lung cancer patients. J Cancer. 2019;10(7):1651-62.

74. Simon-Soro A, Sohn MB, McGinniss JE, Imai I, Brown MC, Knecht VR, et al. Upper respiratory dysbiosis with a facultativedominated ecotype in advanced lung disease and dynamic change after lung transplant. Ann Am Thorac Soc. 2019;16(11):1383-91.

Publisher's Note Springer Nature remains neutral with regard to jurisdictional claims in published maps and institutional affiliations. 